\title{
Potential Therapeutic Effects of Physical Exercise for Bipolar Disorder
}

Alberto Souza de Sá Filho; Antonio Marcos de Souza Moura; Murilo Khede Lamego; Nuno Barbosa Ferreira Rocha; Flávia Paes; Ana Cristina Oliveira; Eduardo Lattari; Ridson Rimes; João Manochio; Henning Budde; Mirko Wegner; Gioia Mura; Oscar Arias-Carrión; Elie Cheniaux; TiFei Yuan; Antonio Egidio Nardi; Sergio Machado

\begin{abstract}
Cognitive deficits are observed in a variety of domains in patients with bipolar disorder (BD). These deficits are attributed to neurobiological, functional and structural brain factors, particularly in prefrontal cortex. Furthermore, cortical alterations in each phase (mania/hypomania, euthymia and depression) are also present. A growing basis of evidence supports aerobic exercise as an alternative treatment method for BD symptoms. Its benefits for physical health in healthy subjects and some psychiatric disorders are fairly established; however evidence directly addressed to BD is scant. Lack of methodological consistency, mainly related to exercise, makes it difficult accuracy and extrapolation of the results. Nevertheless, mechanisms related to BD physiopathology, such as hormonal and neurotransmitters alterations and mainly related to brain-derived neurotrophic factors (BDNF) can be explored. BDNF, specially, have a large influence on brain ability and its gene expression is highly responsive to aerobic exercise. Moreover, aerobic exercise trough BDNF may induce chronic stress suppression, commonly observed in patients with $\mathrm{BD}$, and reduce deleterious effects caused by allostatic loads. Therefore, it is prudent to propose that aerobic exercise plays an important role in $\mathrm{BD}$ physiopathological mechanisms and it is a new way for the treatment for this and others psychiatric disorders.
\end{abstract}

\section{Keywords}

Bipolar disorder, brain-derived neurotrophic factor, depression, exercise, mania, neuroplasticity.

\section{INTRODUCTION}

Bipolar disorder (BD) affects 1 to $1.5 \%$ of the world population [1] and consists of recurrent manic and depressive episodes, interspersed with periods of euthymic mood [2]. BD patients exhibit cognitive dysfunction in a variety of domains, even during periods of clinical remission $[3,4]$. In this sense, the prefrontal cortex (PFC) is an important part of the pathophysiology of $B D$, and has a leading role on several cognitive functions $[5,6]$. Such functions have significant impairments that correlate negatively with social and occupational adjustment [3]. Its chronicity has been consistently attributed to functional and neuroanatomical changes in the cortex that occurs in different magnitudes [5, 7-10]. Given this scenario, the literature starts speculating that programs of physical exercise could act as an important adjuvant and no pharmacological strategy for the treatment of patients with BD [11-14]. Physical exercise, specially aerobic exercise, is recognized for modulate an affective behavior in healthy subjects in moderate intensities of effort [15, 16], modify cerebral activity [17], promote neurogenesis [18], and improve cognitive function [15]. Despite little consistent, significant reduction of symptoms are found with the practice of aerobic exercise in bipolar patients [12,19-21], and its effects related to exercise begin to be established in the literature [13]. Ng et al. [12], for example, observed in 
24 patients diagnosed with $B D$, inserted in a walking group (effort not controlled), an improvement in the Depression Anxiety and Stress Scale (DASS $-p=0.005$ ) and all its subscales (Depression $p=0,048$, Anxiety $p=0,002$, and Stress $p=0,01$ ), compared to 74 bipolar patients who not participated in walking group. In this sense, mechanisms sustaining improvement and insertion of aerobic exercise in lifestyle of patients with BD would be related to alterations in hormonal responses [22], neurotransmitters, such as monoamines, and physiological biomarkers produced by the brain, such as the brain-derived neurotrophic factor (BDNF) and glial cell-derived neurotrophic factor (GDNF) [22, 23]. Although understanding these mechanisms, the evidence still lacks of consistent data that supports the link between remission and BD symptoms to programs of aerobic exercise. Thus, this review paper aims to show the experimental advances of aerobic exercise that can become available as clinical applications in the coming years for bipolar disorder. The literature search was conducted using the databases PubMed, ISI Web of Knowledge and PsycInfo using the following terms and their combinations: "aerobic exercise", "bipolar disorder", "mechanism", "manic/hippomanic", "depressive", "electroencephalogram", "functional magnetic resonance imaging", "cognitive function", "biomarkers". All articles were published between 1980 and 2015 and in English. Additional references were identified through hand search of the possessed articles. Due to the lack of randomized clinical trials on the issue, we decided to select any study, i.e., open and controlled studies, case reports, and cohort and observational studies.

\section{EFFECTS OF AEROBIC EXERCISE ON BIPOLAR DISORDER}

\section{Benefits and/or General Adaptations of the Exercise for Health Promotion - Initial Contextualization}

The American College of Sports Medicine (ACSM) recommends an accumulation of at least 150 minutes of moderate aerobic activity (50-55\% VO2 of reserve - VO2R) weekly or 60 minutes of vigorous activity (70-75\% VO2R) weekly, for health promotion. The result of this summation is observed, primarily, in physical, metabolic and systemic state, providing protection over risk factors associated to coronary artery disease (lipid profile, body mass control, changes in resting glucose and blood pressure) [24]. It is also known that an increase of only 1 MET (1 metabolic equivalent $=3,5 \mathrm{~mL} / \mathrm{kg} / \mathrm{min}$ ) in cardiorespiratory fitness (VO2Max) represents an decrease in relative in mortality of about $13 \%$, independent of any type of disease previously installed, such as coronary artery disease (CAD) $[25,26]$. In addition to physical health, mental health aspects should also be emphasized 22, 25, 74]. The promotion of morphological and functinal changes in several brain areas (PFC, hippocampus) [27], and the formation of new neuronal synapses [28] may reduce the deleterious effects to the brain associated to age and different psychiatric disorders. In this sense, evidence tends to demonstrate positive effects on reducing the severity of symptoms related to illnesses such as $\operatorname{BD}[12,20,21,29]$. Moreover, significant positive modifications in mood with the practice of aerobic exercises $[15,20]$ and the cognitive function [27] are considered, and these benefits are observed in both healthy subjects and in patients with different psychiatric disorders. Bipolar patients, regardless of their clinical status, have low cardiorespiratory fitness and resistance exercise [13], as well, diverse deleterious effects to functional and structural ability of the brain $[3,4,6,8,10,23,30-34]$. Therefore, it is prudent to propose that cardiorespiratory conditioning, that is, improvement of VO2Max is an important pathway for the treatment of different psychiatric disorders, given that their physiopathological mechanisms can be minimized and/or reorganized.

\section{Relationship Between Exercise and Bipolar Disorder}


The current state of art exposes results that confront the real benefit of controlling bipolar illness with programs of aerobic exercises [12-14, 16, 19-21, 27, 35-40]. Few studies were published about this particular topic and these fail to methodological controlling the relation of "dose $x$ response" and/or in several times, there isn't any control. This is a factor that makes the results inconsistent and impossible for future comparisons. For example, $\mathrm{Ng}$, Dodd and Berk [12] evaluated the effects of walking exercise on acute treatment of BD. Twenty four participants diagnosed with $B D$ joined to the program and 74 not participants with the same psychiatric profile were compared. The main results showed a significant reduction on BD symptoms evaluated with Depression Anxiety Stress Scales (DASS; mean \pm standard deviation of participants; $58.2 \pm 25.4>23.0 \pm 14.9$ ). These results should be analyzed careful as well as those reported by Van Citters et al. [39]. The authors produced a pilot study using a specific program of diet reeducation, social behavior and fitness, called SHAPE, with different psychiatric disorders. Within this program, participants performed aerobic activities such as swimming and walking, or strength training or yoga. The criteria of training duration and intensity were adjusted according to an initial observation of the participant's physical state and evaluation scales of psychiatric progress were carried out. Reduction of the severity of symptoms, such as depression, was an important result to be noted $(p=0.003)$ after 9 months follow up, in addition to benefits related to comorbidity in these patients. Other satisfactory results with effect sizes varying from moderate to high were found in different mania and depression scales and an improvement (mean \%) of BD symptoms (MADRS - Montgomery Asberg Depression Scale (23\%); YMRS - Young Mania Rating Scale (27\%) ; CGI Mania - Clinical Global Impression Mania Subscale (43\%); CGI Depression - Clinical Global Impression Depression Subscale (22\%); CGI Overall - Clinical Global Impression Overall Bipolar Illness (-16\%); LIFE-RIFT - Longitudinal Interval Range Impaired Functioning Tool (- 22\%)) [38].

In contrast, a meta-analysis of Pearsall et al. [21] summarizes the possible effects obtained with exercise in a modest and not significant response over the physical activity level in patients with psychiatric disorders, such as bipolar, without effects on negative symptoms of depression $(p=$ 0.43 ) and anxiety $(p=0.14)$. These results should be interpreted carefully given that the control of the relation "dose $x$ response" is questioned in almost all studies. Moreover, and inadequate stimulus may tend to extremes, or to a result without effects in BD symptoms, or the stimulus may be interpreted as an stressor agent, contributing to the relapse of the symptoms [41]. An high intensity of exercise, though still uncertain, seems to be associated to a positive association with $\mathrm{BD}$ symptoms [42] and more precisely, to a negative effect on mood [43]. Contextualizing this data, after performing aerobic exercise to exhaustion, cortisol concentrations remain high for hours while BDNF concentrations are reduced to baseline values in a small amount of time [22] (Table 1). 
Table 1. Papers that investigated the chronic effect of the exercise in patients with bipolar disorder.

\begin{tabular}{|c|c|c|c|c|c|c|c|c|c|c|c|c|c|c|c|c|c|c|c|}
\hline \multirow{3}{*}{ Acticle } & \multicolumn{3}{|c|}{ Gemofral Features } & \multicolumn{7}{|c|}{ Sample Foatures } & \multicolumn{5}{|c|}{ Exercive Features } & \multirow{2}{*}{\multicolumn{4}{|c|}{ Results }} \\
\hline & Comprod & Random & Evalurtion & Patient. & $n$ & Goup & se & Gender & Med & Instrumen & & Enercise & & Times a & Follow. & & & & \\
\hline & & & & & & & & Wrater & & timsuruente & Modality & Time & Intensity & wesk & up & Instrinment & Absolut & $\Delta$ & ES \\
\hline \multirow{10}{*}{$\begin{array}{l}\text { Ng, Dood } \\
\text { \& Berk, [12 }\end{array}$} & \multirow{10}{*}{ 12] Yos } & \multirow{10}{*}{$\mathrm{N}$} & \multirow{10}{*}{$(\mathrm{COD} \cdot 10)$} & \multirow{10}{*}{$\begin{array}{l}\text { Bipdar } \\
\text { (100\%) }\end{array}$} & & & & & & \multirow{10}{*}{ 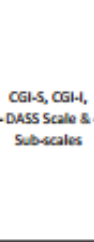 } & & & & & \multirow{10}{*}{${ }_{\text {manth }}^{24}$} & CG1.5 & $4.2 \pm 1.0>2.5 \pm 1.2$ & $40.5 \mathrm{~m}$ & 1.7 \\
\hline & & & & & & & & & & & & & & & & CGH. & Non-reported & . & . \\
\hline & & & & & 14 & Exorcke & $\begin{array}{l}43.6 \\
(15.0)\end{array}$ & $M+F$ & ? & & Walk & $40 \mathrm{~min}$ & Frow & ? & & DASS Depres & $240 \pm 12.4 \times 7.2 \pm 7.3$ & $70.0 \mathrm{~s}^{\circ}$ & 14 \\
\hline & & & & & & & & & & & & & & & & Dass Stress & $12.4 \pm 12.3 \times 9.2 \pm 7.2$ & $52.6 \mathrm{~N}^{*}$ & 0.8 \\
\hline & & & & & & & & & & & & & & & & DASS Anviety & $14.8 \pm 112 \times 6.6 \pm 4.5$ & $55.4 \mathrm{~N}^{*}$ & 0.7 \\
\hline & & & & & \multirow{5}{*}{35} & \multirow{5}{*}{$\begin{array}{l}\text { Non- } \\
\text { eworchese }\end{array}$} & \multirow{5}{*}{$\begin{array}{l}43.9 \\
\text { (18.5) }\end{array}$} & \multirow{5}{*}{$M+F$} & \multirow{5}{*}{ ? } & & \multirow{5}{*}{. } & \multirow{5}{*}{. } & \multirow{5}{*}{$\cdot$} & \multirow{5}{*}{ - } & & CGl-5 & $4.3 \pm 0.8 \times 2,4 \pm 0.8$ & $44.2 \%$ & 2.4 \\
\hline & & & & & & & & & & & & & & & & CGL-1 & Non-reperted & - & - \\
\hline & & & & & & & & & & & & & & & & DASS Depres & $27.3 \pm 12.3>13.7 \pm 12.8$ & $49.88 \mathrm{~K}$ & 1.1 \\
\hline & & & & & & & & & & & & & & & & DASS Stress & $25.3 \pm 9.3>17.1 \pm 11.6$ & 32456 & 0.9 \\
\hline & & & & & & & & & & & & & & & & DASS Aecóety & $18.2 \pm 8.9>13.8 \pm 8.9$ & 2425 & 0.5 \\
\hline \multirow{5}{*}{$\begin{array}{c}\text { Van } \\
\text { Caters, at } \\
\text { a. [39] }\end{array}$} & & & & & & & & & & & & & & & & SF-12 MCS & $32.2 \pm 12.0 \times 36.4 \pm 13.4$ & $13.0 \mathrm{~s}^{*}$ & 0,30 \\
\hline & & & & & & & & & & $\begin{array}{l}\text { (5F-12 MCS) } \\
\text { (5F-12 PCS) }\end{array}$ & & & & & & SF-12 PCS & $44.7 \pm 12.5>44.3 \pm 11.1$ & $0.9 \mathrm{\kappa}$ & 0 \\
\hline & Not & & & (1992) & 76 & Diet Relax & {$[11.4)$} & $\mathrm{M} * \mathrm{~F}$ & ? & CESD, RSES & Swimming & $\min$ & Frow & times & manth & CESD & $28.5 \pm 13.0 \times 26.0 \pm 15.4$ & $9.1 \mathrm{\kappa}$ & 0.2 \\
\hline & & Not DSR & SM.N & & & & & & & SANS & & & & & & GSES & $53.3 \pm 18.5 \times 57.4 \pm 18.4$ & $7.7 \%$ & 0.2 \\
\hline & & & & & & & & & & & & & & & & SANS & $24 \pm 0.7>2.3 \pm 0.5$ & $4.2 \mathrm{*}^{*}$ & 0.1 \\
\hline & & & & & & & & & & & & & & & & MADES & $17.2 \pm 5.2 \times 13.2 \pm 10.1$ & $23.3 \mathrm{~N}^{*}$ & 0.8 \\
\hline & & & & & & Eworcke & & & & & & & Moderate & & & YMPS & $4.4 \pm 2.0 \times 5.6 \pm 3.9$ & $27.3 \mathrm{~s}^{*}$ & 0.6 \\
\hline Sphia, of & Nor & Net & Mina-Plus & Bipolar & 5 & LWestyle & 440 & $M+F$ & $?$ & $\begin{array}{l}\text { YMPS } \\
\text { YCG }\end{array}$ & $?$ & $30 \mathrm{~min}$ & Effort (X) & 5 times: & 5 moeth & CGLEP M & $14 \pm 0.9 \times 2.0 \pm 0.7$ & $42.9 \mathrm{~N}^{\circ}$ & 0.6 \\
\hline 2.. [38] & noot & Not & CGLBP & (1000) & & Charges & (16.0) & Mrt & $r$ & D, o & & & non- & & & CGI-EP D & $3.6 \pm 0.6>2.8 \pm 1.3$ & $22.2 \mathbf{w}^{*}$ & 1.3 \\
\hline & & & & & & Work & & & & (LFF-9uFT) & & & reportestest & & & CGLBP O & $3.8 \pm 0.5>3.2 \pm 0.8$ & $15.8 \mathrm{x}^{*}$ & 1.2 \\
\hline & & & & & & & & & & & & & & & & UFE- 6 FI T & $12.0 \pm 3.1>9.4 \pm 2.1$ & $21.7 \mathbf{x}^{*}$ & 0.8 \\
\hline & & & & & & Eworcke & & & & & & & & & & SF36 PCS & $40.4 \pm 7.7 \times 39.4 \pm 7.5$ & $2.5 \%$ & 0.1 \\
\hline & & & & & 123 & Social & (12.5) & $M+F$ & Yes & & Walk & $30 \mathrm{~min}$ & EMart & 3 times & & SF 35 MCS & $35.6 \pm 8.7 \times 34.8 \pm 7.9$ & 2.28 & 0.1 \\
\hline Vertusegh & Yes & Yes & DSM-N & Eipolar - & & Copnitive & & & & (SF $35 \mathrm{MCS})$ & & & & & clenth. & BSI PST & $27.6 \pm 12.6 \times 25.3 \pm 12.8$ & 8.36 & 0.2 \\
\hline$\therefore$ & & & & (24): & & & 46.6 & & & (BSI PST) & & & & & 6 moeth & SF36 PCS & $412 \pm 5.9 \times 40.2 \pm 7.8$ & $2.4 \mathrm{~K}$ & 0.2 \\
\hline & & & & & 50 & control & (11.9) & $M+F$ & Yes & & - & - & - & - & & SF 36 MCS & $35.8 \pm 7.9 \times 35.3 \pm 7.4$ & $1.4 \mathrm{~K}$ & 0.1 \\
\hline
\end{tabular}

Subtitles: * - Significant Difference; ES - Effect Size; Med - Medications, ICD-10 - Intermational Statistical Classification of Deseases and Related Health Problems; CGI S - Clinical Global Impression Severity; CGI I - Clinical Global Impression Improvement; DASS - Depression Anxiety Stress Scale; Mental Health Functioning - (SF-12 MCS); Psysical Health Functioning - (SF-12 PCS); Depression - (CESD); Self-Efficacy - (RSES); Negative Symptoms (SANS); MINI-Plus - Mini International Neuropsychiatric Interview; CGI-BP Clinical Impression-Bipolar, MADRS - Montgomery Asberg Depression Rating; YMRS - Young Mania Rating Scale; CGI-BP M - Clinical Impression-Bipolar Mania; CGil-BP D Clinical Impression-Bipolar Depression; CGi-BP O - Clinical Impression-Bipolar Overall; LIFE-RIFT - Longitudinal Interval Follow-up Evaluation Range Impaired Functioning Tool; SF36 PCS - Physical component score; SF 36 MCS - Mental component score; BSI PST - Brief Symptom Inventory Positive Symptom Total.

Possible Exercise Mechanisms that Influence Bipolar Disorder Despite of inconsistencies and limitations, there is a tendency to obtain positive results with exercise practice $[11,12,16,36$, $38,39]$. The improvement mechanism of the severity of BD symptoms may be connected to stress reduction from physiopathology agent's characteristics of the illness. Chronic stress caused by the imbalance between proinflammatory cytokines, changes in corticosteroid and reduction of BDNF levels, create a propitious ambient to increased allostatic charges and consequent reduction of cerebral neuroplasticity [19]. Therefore, subjects that practice appropriated periods of exercise (threshold around $20-30 \mathrm{~min}$ ) may produce appropriated results on anxiety and reduction of stress reactivity in long term [43]. Chronic stress is also suggested as the most precipitator of mood disorders, given that a great sensitivity to stress is related to recurrent episodes [44]. This fact can be explained also by a defective modulation of monoaminergic systems $[45,46]$, such as observed in different psychiatric disorders. Several theories support a possible influence of mechanisms of dopaminergic transport [46] or GABAergic influence on physiopathological characteristics of BD [19]. This last, in special, has an important influence on gene expression of BDNF by hippocampus during exercise, influencing directly the basal levels of this substance [19]. During acute exercise of mild to moderate effort, cortisol and BDNF concentrations was not significantly altered, but not for high intensities [22]. High cortisol concentration is normally co-regulated by BDNF concentration and both follow a linear increase pattern [47]. However, in bipolar patients, initial BDNF concentration is normally depressed [23] which may mitigate the co-regulator and protection effect of BNDF. Despite of acutely after exercise the BDNF concentrations come back closely to baseline values [22], chronically the main benefits on symptoms severity are primarily associated to basal BNDF concentrations. As explained, high basal concentrations of this substance are deeply connected to neuroplasticity and cerebral neurogenic capacity $[11,19,40]$ and these responses extend

to different BD domains, including cognition [35]. Exercise effects on cerebral neurotrophins were demonstrated by Seifert et al. [37] in a cycling training program aiming $600 \mathrm{kcal} / \mathrm{day}( \pm 70 \%$ 
FC maximum/ \pm 60 min of exercise), during 3 months. The authors observed significant increases in arterial BDNF concentration in rest (baseline - $58 \pm 106$; follow-up - $206 \pm 108 \mathrm{ng} .100 \mathrm{~g}$-1.min$1)$, for the training group $(p<0.05)$. The Control group was included just on a diet program (similar energy cost of exercise), but not reaching significant improvement. Exercise acts increasing gene expression of BDNF and messenger RNA in diverse body cells, including in muscular cells, but mainly in the hippocampal region [19].

Regarding cognition, BDNF can be an important key to cognitive recovery $[18,48]$. The mechanism is associated mainly to synaptic plasticity changes, namely, better transmission and/or new synaptic connections [28]. It is demonstrated possible long term synaptic potentiating localized in hippocampal area, which is traduced in a better memory $[18,48]$. Evidences suggest that effects produced by the relation between BDNF and new neurons formation may favor cognitive domains of adult learning [49]. Pharmacological administration helps maintaining and/or reducing cognitive deficit on bipolar subjects [50]. In these patients, treatment with lithium is the first treatment option and has significant effects on increasing volume of amygdala and hippocampus [51], neuroanatomical structures fundamental for cognitive performance and affective state [50]. Aerobic exercise seems to have similar effect on neuroanatomical organization and cognitive function in healthy subjects [27] and in different psychiatric disorders $[52,53]$. Despite this understanding, little is known about aerobic exercise influence on different cognitive domains of BD. Exercise is recognized for inducing an increase in cerebral volume and consequently is associated to cognitive function changes. Colcombe et al. [27] implemented tree weekly sessions of 60 minutes training, initiating at $40-50 \%$ of heart rate reserve (HRR) and progressing along six months to prescribed intensities of $60-70 \%$ of HRR. The authors reported significant increases, mainly in white and grey matters, localized at PFC (right inferior frontal gyrus $-p \leq 0.05$ ) and temporal cortex (left superior temporal gyrus $-p$ $\leq 0.05$ ). PFC region plays an important role in BD physiopathology and in motor control, attention and executive function [8]. Thus, larger changes in cerebral volume can bring important cognitive gains in long term.

\section{CONCLUSION}

Strong evidence in literature suggests using aerobic exercise as an important prophylactic strategy of actuation similar to pharmacology, aiming a reduction on symptoms severity and BD treatment in long term. Neurotrophins, mainly BDNF, obtained increasing relevance in what concerns aerobic exercise benefits. Complementary studies should be applied using different exercise protocols and controlled "dose $\mathrm{x}$ response" to evaluate better strategies for the symptoms treatment. Despite possible positive responses to exercise on BD symptoms, results still remain inconclusive and should be interpreted and implemented carefully.

\section{LIST OF ABBREVIATIONS}

ACSM = American College of Sports Medicine

$\mathrm{BD}=$ Bipolar Disorder

BDNF = Brain-Derived Neurotrophic Factor

$\mathrm{CHD}=$ Coronary Heart Disease

DASS $=$ Depression Anxiety and Stress 
$\mathrm{ES}=$ Effect Size

$\mathrm{fMRI}=$ Functional Magnetic Resonance Imaging

GDNF = Glial Cell-Derived Neurotrophic Factor

PFC $=$ Prefrontal Cortex

VO2Max = Maximal Oxygen Consumption

\section{REFERENCES}

[1] Bebbington P, Ramana R. The epidemiology of bipolar affective disorder. Soc Psychiatry Psychiatr Epidemiol 1995; 306: 279-92.

[2] Belmaker RH. Bipolar disorder. N Engl J Med 2004; 3515: 476-86.

[3] Martinez-Aran A, Vieta E, Reinares M, et al. Cognitive function across manic or hypomanic, depressed, and euthymic states in bipolar disorder. Am J Psychiatry 2004; 1612: 262-70.

[4] Robinson LJ, Ferrier IN. Evolution of cognitive impairment in bipolar disorder: a systematic review of cross-sectional evidence. Bipolar Disord 2006; 82: 103-16.

[5] Adler CM, Holland SK, Schmithorst V, Wilke M, Weiss KL, Pan H, et al. Abnormal frontal white matter tracts in bipolar disorder: a diffusion tensor imaging study. Bipolar Disord 2004; 63: 197203.

[6] Osuji IJ, Cullum CM. Cognition in bipolar disorder. Psychiatr Clin North Am 2005; 282: 42741.

[7] McIntosh AM, Whalley HC, McKirdy J, et al. Prefrontal function and activation in bipolar disorder and schizophrenia. Am J Psychiatry 2008; 1653: 378-84.

[8] Strakowski SM, DelBello MP, Sax KW, et al. Brain magnetic resonance imaging of structural abnormalities in bipolar disorder. Arch Gen Psychiatry 1999; 563: 254-60.

[9] Blumberg HP, Leung HC, Skudlarski P, et al. A functional magnetic resonance imaging study of bipolar disorder: state- and trait-related dysfunction in ventral prefrontal cortices. Arch Gen Psychiatry 2003; 606: 601-9.

[10] Adler CM, Holland SK, Schmithorst V, Tuchfarber MJStrakowski SM. Changes in neuronal activation in patients with bipolar disorder during performance of a working memory task. Bipolar Disord 2004; 66: 540-9.

[11] Alsuwaidan MT, Kucyi A, Law CWMcIntyre RS. Exercise and bipolar disorder: a review of neurobiological mediators. Neuromol Med 2009; 114: 328-36.

[12] Ng F, Dodd SBerk M. The effects of physical activity in the acute treatment of bipolar disorder: a pilot study. J Affect Disord 2007; 1011-3: 259-62.

[13] Shah A, Alshaher M, Dawn B, et al. Exercise tolerance is reduced in bipolar illness. J Affect Disord 2007; 1041-3: 191-5.

[14] Fagiolini A, Frank E, Scott JA, Turkin SKupfer DJ. Metabolic syndrome in bipolar disorder: findings from the Bipolar Disorder Center for Pennsylvanians. Bipolar Disord 2005; 75: 424-30. 
[15] Tomporowski PD. Effects of acute bouts of exercise on cognition. Acta Psychol 2003; 1123: 297-324.

[16] Sylvia LG, Ametrano RMNierenberg AA. Exercise treatment for bipolar disorder: potential mechanisms of action mediated through increased neurogenesis and decreased allostatic load. Psychother Psychosom 2010; 792: 87-96.

[17] Brummer V, Schneider S, Abel T, Vogt TStruder HK. Brain cortical activity is influenced by exercise mode and intensity. Med Sci Sports Exerc 2011; 4310: 1863-72.

[18] van Praag H, Schinder AF, Christie BR, Toni N, Palmer TDGage FH. Functional neurogenesis in the adult hippocampus. Nature 2002; 4156875: 1030-4.

[19] Cotman CW, Berchtold NC. Exercise: a behavioral intervention to enhance brain health and plasticity. Trends Neurosci 2002; 256: 295-301.

[20] Daumit GL, Dickerson FBAppel L. Weight loss in persons with serious mental illness. N Engl J Med 2013; 3695: 486-7.

[21] Pearsall R, Smith DJ, Pelosi AGeddes J. Exercise therapy in adults with serious mental illness: a systematic review and meta-analysis. BMC Psychiatry 2014; 141: 117.

[22] Rojas Vega S, Struder HK, Vera Wahrmann B, Schmidt A, Bloch WHollmann W. Acute BDNF and cortisol response to low intensity exercise and following ramp incremental exercise to exhaustion in humans. Brain Res 2006; 11211: 59-65.

[23] Cunha $A B$, Frey BN, Andreazza AC, et al. Serum brain-derived neurotrophic factor is decreased in bipolar disorder during depressive and manic episodes. Neurosci Lett 2006; 3983: 215-9.

[24] Swain DP, Franklin BA. Comparison of cardioprotective benefits of vigorous versus moderate intensity aerobic exercise. Am J Cardiol 2006; 971: 141-7.

[25] Myers J, Prakash M, Froelicher V, Do D, Partington SAtwood JE. Exercise capacity and mortality among men referred for exercise testing. N Engl J Med 2002; 34611: 793-801.

[26] Kodama S, Saito K, Tanaka S, et al. Cardiorespiratory fitness as a quantitative predictor of all-cause mortality and cardiovascular events in healthy men and women: a meta-analysis. JAMA 2009; 30119: 2024-35.

[27] Colcombe SJ, Erickson KI, Scalf PE, et al. Aerobic exercise training increases brain volume in aging humans. J Gerontol A Biol Sci Med Sci 2006; 6111: 1166-70.

[28] Schinder AF, Poo M. The neurotrophin hypothesis for synaptic plasticity. Trends Neurosci 2000; 2312: 639-45.

[29] Pearsall R, Hughes S, Geddes JPelosi A. Understanding the problems developing a healthy living programme in patients with serious mental illness: a qualitative study. BMC Psychiatry $2014 ; 1438$.

[30] Anticevic A, Brumbaugh MS, Winkler AM, et al. Global prefrontal and fronto-amygdala dysconnectivity in bipolar I disorder with psychosis history. Biol Psychiatry 2013; 736: 565-73.

[31] Blumberg HP, Kaufman J, Martin A, et al. Amygdala and hippocampal volumes in adolescents and adults with bipolar disorder. Arch Gen Psychiatry 2003; 6012: 1201-8. 
[32] Chang K, Adleman NE, Dienes K, Simeonova DI, Menon VReiss A. Anomalous prefrontalsubcortical activation in familial pediatric bipolar disorder: a functional magnetic resonance imaging investigation. Arch Gen Psychiatry 2004; 618: 781-92.

[33] Barbosa IG, Huguet RB, Sousa LP, et al. Circulating levels of GDNF in bipolar disorder. Neurosci Lett 2011; 5022: 103-6.

[34] Kunz M, Cereser KM, Goi PD, et al. Serum levels of IL-6, IL-10 and TNF-alpha in patients with bipolar disorder and schizophrenia: differences in pro- and anti-inflammatory balance. Rev Bras Psiquiatr 2011; 333: 268-74.

[35] Knaepen K, Goekint M, Heyman EMMeeusen R. Neuroplasticity - exercise-induced response of peripheral brain-derived neurotrophic factor: a systematic review of experimental studies in human subjects. Sports Med 2010; 409: 765-801.

[36] Matta Mello Portugal E, Cevada T, Sobral Monteiro-Junior R, et al. Neuroscience of exercise: from neurobiology mechanisms to mental health. Neuropsychobiology 2013; 681: 1-14.

[37] Seifert T, Brassard $P$, Wissenberg $M$, et al. Endurance training enhances BDNF release from the human brain. Am J Physiol Regul Integr Comp Physiol 2010; 2982: R372-7.

[38] Sylvia LG, Salcedo S, Bernstein EE, Baek JH, Nierenberg AADeckersbach T. Nutrition, Exercise, and Wellness Treatment in bipolar disorder: proof of concept for a consolidated intervention. Int J Bipolar Disord 2013; 11: 24.

[39] Van Citters AD, Pratt SI, Jue K, et al. A pilot evaluation of the In SHAPE individualized health promotion intervention for adults with mental illness. Community Ment Health J 2010; 466: 54052.

[40] van Praag H, Christie BR, Sejnowski TJGage FH. Running enhances neurogenesis, learning, and long-term potentiation in mice. Proc Natl Acad Sci USA 1999; 9623: 13427-31.

[41] Hammen C, Gitlin M. Stress reactivity in bipolar patients and its relation to prior history of disorder. Am J Psychiatry 1997; 1546: 856-7.

[42] Dakwar E, Blanco C, Lin KH, Liu SM, Warden D, Trivedi M, et al. Exercise and mental illness: results from the National Epidemiologic Survey on Alcohol and Related Conditions (NESARC). J Clin Psychiatry 2012; 737: 960-6.

[43] Ekkekakis P, Petruzzello SJ. Acute aerobic exercise and affect: current status, problems and prospects regarding dose-response. Sports Med 1999; 285: 337-74.

[44] Dienes KA, Hammen C, Henry RM, Cohen ANDaley SE. The stress sensitization hypothesis: understanding the course of bipolar disorder. J Affect Disord 2006; 951-3: 43-9.

[45] Berk M, Dodd S, Kauer-Sant'anna M, et al. Dopamine dysregulation syndrome: implications for a dopamine hypothesis of bipolar disorder. Acta Psychiatr Scand Suppl 2007; 434: 41-9.

[46] Greenwood TA, Alexander M, Keck PE, et al. Evidence for linkage disequilibrium between the dopamine transporter and bipolar disorder. Am J Med Genet 2001; 1052: 145-51.

[47] Begliuomini S, Lenzi E, Ninni F, et al. Plasma brain-derived neurotrophic factor daily variations in men: correlation with cortisol circadian rhythm. J Endocrinol 2008; 1972: 429-35. 
[48] Schinder AF, Gage FH. A hypothesis about the role of adult neurogenesis in hippocampal function. Physiology 2004; 19253-61.

[49] Leuner B, Gould EShors TJ. Is there a link between adult neurogenesis and learning? Hippocampus 2006; 163: 216-24.

[50] Hajek T, Kopecek M, Hoschl CAlda M. Smaller hippocampal volumes in patients with bipolar disorder are masked by exposure to lithium: a meta-analysis. J Psychiatry Neurosci 2012; 375: 333- 43.

[51] Foland LC, Altshuler LL, Sugar CA, et al. Increased volume of the amygdala and hippocampus in bipolar patients treated with lithium. Neuroreport 2008; 192: 221-4.

[52] Fremont J, Craighead LW. Aerobic Exercise and Cognitive Therapy in the Treatment of Dysphoric Moods. Cogn Ther Res 1987; 112: 241-51.

[53] Kubesch S, Bretschneider V, Freudenmann R, et al. Aerobic endurance exercise improves executive functions in depressed patients. J Clin Psychiatry 2003; 649: 1005-12. 